\title{
Chapter 14: The challenge of performing research which will contribute helpful engineering knowledge concerning emergence
}

Author(s):

Timothy L.J. Ferris

Cranfield University, Defence Academy of the United Kingdom Shrivenham, SN6 8LA, timothy.ferris@cranfield.ac.uk

\section{Chapter Abstract}

This chapter discusses the nature of research which is required to provide knowledge of emergence that is useful to engineers in the conduct of engineering work. The discussion begins with three elements which form the foundation of the argument: the contrast of the purposes of engineering and science and the different perspectives of knowledge associated with those purposes; the diversity of views on "emergence" and an argument for working with one definition; and an outline of a framework for classifying the objectives of research activities associated with the kind of knowledge that is needed. These foundations are used to evaluate a number of approaches to research in engineering to assess the contribution that the research approaches make to discovery of matters associated with emergence. The research approaches considered are case studies, forensic investigations, post hoc and experimental studies, fundamental theoretical studies and contributions to the engineering task. These approaches instantiate different research purposes and therefore, naturally will be associated with different methods. The previous analysis is discussed in the context of the nature of research published in response to the imperatives presented to faculty by the career measures of success to explain the current emphasis on what is investigated and the limitations for engineering in that focus. In the 
final section an approach for addressing the issue of emergence in engineering is presented which leads to a brief statement of a desirable outcome of research into emergence conducted with a view to assisting the practice of engineering.

\subsection{Introduction: Knowledge in Engineering}

"Engineering is not a science, but a practice of the necessary to achieve a given goal" (Cripps 1991, p. 16). This quotation summarizing the nature of engineering is important in framing the nature and role of knowledge, and therefore of research, in engineering. In turn, the pragmatic nature of engineering specifically leads to a particular kind of knowledge of emergence being necessary in order to be useful. The issue of kinds of knowledge will be discussed throughout this chapter.

The task of engineering is to bring into being products and systems which provide, in a desirable manner, effective solutions to needs or means to produce a required effect. The purposed of science is to develop knowledge which is tested to provide confidence in the truth of the knowledge, which is commonly formulated as the testing of hypotheses. In this process, a scientist makes general observations of an area of interest which enable the generation of an hypothesis that becomes the subject of a test, and through that testing knowledge of the phenomenon relevant to the hypothesis is produced. During the 1950 s there was a general trend by engineers to assert the scientific credentials of engineering (Ferris 2007a, Ferris 2007b, Ferris 2008). This followed the significant engineering advances made during World War II that were clearly associated with the application of scientific knowledge in the development of technologies which contributed significantly to the conduct and outcome of the war. The associated factor was that "science" was the recently emerged criterion for admission of a field of study into universities, so it was felt necessary for engineers to demonstrate the scientific 
credentials of engineering. At the extreme of this trend Hickey wrongly proceeded to assert that an engineer has an hypothesis which can only be tested through implementation in a product (Hickey 1960, p. 74). However, the engineer's goal, qua engineer, is not to test an hypothesis related to the possibility of developing a solution, but rather to provide the most appropriate solution for the need consistent with Cripps (1991).

The popular hendiadys "science and engineering" conflates and confounds engineering and science, treating them as part of the same enterprise, whereas they are distinct. Science seeks to generate knowledge about the world and things and phenomena within it. To this end science has developed methods of discovery which instantiate application of the view of knowledge and learning provided in the traditional western epistemology. The classical epistemological account of knowledge is expressed in the trifold conditional statement, or a variation:

"S knows that $p$ if and only if:

1. $\mathrm{p}$ is true;

2. $\mathrm{S}$ believes that $\mathrm{p}$; and

3. S is is justified in believing that p" (Gettier 1967).

Gettier's paper is very controversial because he challenged this account of the requisites of knowledge and several variants but, in turn, many epistemologists have objected to his view. Engineering seeks to provide appropriate and useful outcomes. Thus, engineers use the best available knowledge about phenomena which they intend to exploit, including, and often predominantly, the knowledge developed through science, to predict results expected from design configuration which are posited as candidate solutions. In the acronym STEM (Science Technology Engineering and Mathematics) the science and engineering hendiadys is augmented by "technology" and "mathematics". Mathematics is used in engineering analysis and in 
engineering research, often to the extent that engineering appears like a branch of applied mathematics when, really, the power of mathematics in engineering follows because of the homomorphism of mathematics and the phenomena of real things, and successful engineers always keep in mind the limitations of their analytical models and seek empirical evidence in their work. Engineering, in the use of mathematics to predict expected outcomes of design configurations tests the extent to which the homomorphism assumption is reasonable.

Although engineering research is mathematically intensive, engineering research is fundamentally different than research in mathematics. Research in mathematics is focused on the exploration of the implications of the axioms of a mathematical system whereas physical science research is about the phenomena and the role of mathematics is description of observations and in engineering research the focus is on finding appropriate interventions in the world and the role of mathematics is as a tool to enable prediction of outcomes. A further purpose of mathematics in engineering research is to develop design tools to determine the parameters of design configurations that exploit known phenomena to achieve specified measures of performance. In summary, knowledge in engineering concerns the subject matter of things that are useful in the engineering of solutions to needs and the methods required to apply the scientific knowledge of phenomena to the achievement of solutions to needs.

\subsection{Definition of Emergence}

Engineering is the endeavor in which things are combined according to a design with the purpose of achieving specific effects which are different than the effects or properties of the elements which are arranged through the design and building work. The goal of achieving effects through combining things that do not, by themselves, produce those effects is consistent with the common definition of emergence (Hitchins 1992). In the common definition emergence is the 
phenomenon that results in the effect of a combination of elements that form a system being different than the effect observed from any of the elements of the system taken alone. This juxtaposing of a description of the objectives of engineering and the definition of emergence makes it appear that all engineering work would be recognized as involving emergence, but this is not so universally accepted in the system engineering community, which is the major engineering community in which emergence is discussed.

Discussion of emergence in the systems engineering community may produce a recitation of simplistic statements, such as "the whole is greater than the sum of the parts", or an argument that emergence refers to events that take one by surprise, or even just nasty surprises, sometimes euphemistically referred to as "undocumented features" (BKCASE Editorial Board 2016). None of these approaches is helpful in enabling substantial engagement with the idea of emergence. To assert that "the whole is greater than the sum of the parts" does not help anyone design a system to provide desired services, performance or some other characteristic, and simultaneously not produce either undesired or undesirable outcomes. The major problem with the simplistic statement is that it does not guide the achievement of the desired results.

The second approach is to regard emergence as concerning the things that take one by surprise, that is, the unexpected outcomes of assembling a system. The problem with this view is that it does not have a fixed boundary between what is, in contrast to that which is not, classified as emergent. Thus, a good designer, who knows a lot, may be able to predict what happens in certain circumstances and not be surprised whereas a less knowledgeable engineer may be surprised by the same properties of a system under development. Alternatively, as knowledge develops, effects which were previously unobserved and unexpected have been observed on first occurrence and therefore transition from emergent to known, and presumably, expected 
phenomena. A definition of anything that results in such a fluid scope of that to which the term applies is not helpful. The only place in engineering where such a concept of unexpected and surprising is useful as a separate classification is in legal defense in cases where the argument is whether an appropriately competent engineer should have been aware of, and expected, systemic behaviors that caused a problem.

The third view is that emergence concerns undesirable effects found after assembly of the system. To define emergence as concerned with unexpected undesirable effects trivializes emergence in two ways. First, it associates emergence with the subclass of unpredicted or unpredictable outcomes which are also undesirable. This concern is addressed above. Second, it associates emergence with that which is considered undesirable. This is problematic because in the assembly of a system a particular property may be considered desirable or undesirable as a result of a stakeholder's viewpoint, with different stakeholders judging particular effects in opposite, or at least significantly different, ways.

Each of the three approaches to defining emergence is unsatisfactory because they yield different views concerning what is emergent, differing depending on viewpoint, time or knowledge. Such a definition can do no better than allow, and even encourage, imprecise thought.

A useful definition must:

1. Provide terminology that has constant breadth of inclusion of cases.

2. Name something independently of connotations of value concerning the goodness, or otherwise, of the subject matter.

In this chapter we follow Checkland, as quoted in SEBoK (BKCASE Editorial Board 2016), who defined emergence as "the principle that entities exhibit properties which are meaningful only when attributed to the whole, not to its parts." 
This definition has the desirable characteristics of:

1. Not ascribing a value judgment, good or bad, on anything described as emergent.

2. Providing a time, knowledge and perspective invariant boundary between emergence and not-emergence.

3. Accommodating the primary goal of engineering, to make systems that satisfy needs through assembling components to produce effects that are not achieved by the components taken alone.

Another philosophical issue arises: if one knows certain facts does one also know their logical consequences (Hintikka 1970)? This issue is important because emergence is the consequence of multiple elements, each with its own properties, being assembled into an interconnected construct. The question is whether, in adducing certain facts about the elements of a system, it can be said that one is aware of their consequences and interactions. If one were aware of the interactions one would not be surprised by the effects of the combination of known entities. Experience shows that surprises happen. We conclude that one may be aware of entities and their characteristics but not have immediate awareness of the characteristics of a system constructed from the entities. We also note that design relies on the consistency of matter, so that previous assemblies of entities enable prediction, based on precedence, of the properties that will be observed in future assemblies (Goodman 1973).

\subsection{Framework to Organize Research Objectives}

Research is commonly understood as the process by which new knowledge is created. Each academic field has developed traditions about both the nature of knowledge which is desired and the research methods to develop it. In most disciplines there are one or a few methods which have become accepted as the possible means of developing knowledge and these have become so 
embedded in the culture of the discipline that they are part of the social and intellectual construct of the discipline. The famous workers in the discipline achieved their status through the use of those methods and those aspiring to recognition are effectively bound to pursue the same methods, on pain of ostracism. Those who have succeeded are rarely motivated to challenge the status quo. The consequence is that most disciplines, after an initial establishment phase, move to a continual refinement phase until, perhaps, something happens that results in a paradigm shift (Ackoff 1979a; Ackoff 1979b; Ackoff 2001; Kline 1995).

As we consider the research as the activity that develops knowledge we look to several forms of knowledge which have been identified. The first formulation is "know that", following Ryle's distinction between "knowing that" and "knowing how" (Ryle 1948). This distinction is significant in engineering, where both kinds of knowledge are required (Bucciarelli 2003). "Knowing that" is of the form of declarative knowledge, that is, knowledge of a kind that can be articulated to represent ideas. Knowledge of this type is about things "out there", objects which can be observed, but primarily in an object relation to the knower and speaker. This kind of knowledge is of a form which is relatively easy to teach because it is possible to reduce the teaching to statement of the representation. This is expanded in education in the development of kinds of ability to interact with the content as described in the kinds of learning described in the 3 cognitive domain of Bloom's taxonomy (Bloom et al. 1979). "Know that" is associated with the conceptualization of knowledge as concerning the representations of facts rather than the assimilation of the significance of the facts into an action guiding construct.

The second formulation is "know how", Ryle's formulation to describe capacity to perform a function (Ryle 1948). The capacity to act appropriately is distinct from capacity to describe knowledge related to the function or the relevant theory. Knowing how does not preclude ability 
to articulate what is known, but emphasizes the ability to perform an act. The distinctive feature of knowing how is that the subject's orientation is with doing rather than describing something. "Knowing how" may be expressed in ability to use that which is the subject of "know that" but may also include abilities to perform action. Ability to perform action can be expanded using the psychomotor domain, for example as expanded in engineering (Ferris 2010). The ability to judge what is appropriate to do also involves development of the individual in the affective domain, which is concerned with developing value systems that lead to appropriate action (Krathwohl et al. 1964).

A third kind of knowledge is named "knowing" by Nissen (Nissen 2006) and as "procedural knowledge", or "skill" by Biggs (Biggs 1999). The emphasis in "knowing" is on the ability to choose and perform some action in an appropriate and effective manner. Thus, Nissen says that knowing how to ride a bicycle is demonstrated by mounting and actually riding a bicycle. "Knowing" is tested or demonstrated through a practical test in which the candidate must perform the action in a fluent manner. Ability to articulate anything about the matter, its situation, or a theory about the action or its situation is irrelevant to "knowing". "Knowing" contrasts with "know how". In "know how" the emphasis is on performing a function as a capacity, but "knowing" is usually associated with ability to make sound judgements about the action and whether to act. Thus, knowing is a capability that arises from appropriate and fluent application of that capacity to act.

The three kinds of knowledge relate to abstraction, description and theorization, through applied knowledge which enables performance of action, to ability to perform fluently. The three named kinds of knowledge are identifiable points in characterizing knowledge, but any particular instance of knowledge embodies a particular combination of abstract theorizing, ability to act, 
and fluency of action, thus positioning the knowledge at a particular place in a continuous knowledge space.

Identifying the three kinds of knowledge is useful for theorizing about knowledge and, by extension, research. Research creates new knowledge of some kind. The new knowledge developed through research must be new to everyone, not just new to the investigator. The increment of advance of knowledge may be of any size, possibly very small, and situationally constrained. This contrasts with science which seeks to generate context independent knowledge. But another very important factor is introduced through the recognition of the three kinds of knowledge. This is that research may be performed with a variety of objectives with respect to the kind of knowledge that is to be developed. There are two possible approaches to this challenge of finding an organization of objectives for research. One is to identify the kinds of knowledge produced by activities currently recognized as research. This approach has the problem that it limits the task to description of what has been done already. This author followed this approach, with collaborators, but found it frustrating, as did the collaborators (Ferris et al. 2005; Ferris et al. 2007). The alternative is to look for a complete framework of kinds of knowledge, which could lead to generation of possible objectives of research. We choose the latter approach which has the advantage of holding potential for a greater completeness and seems to overcome the frustration described above.

Augustine of Hippo, in De Civitate Dei XIX:1, refers to Marcus Varro's now lost work, On Philosophy, which presented an analysis of the characteristics of all the actual and possible schools of philosophy (Augustine of Hippo 1984). Varro had organized philosophies according to their position, in a set of two or three categories, in each of six dimensions, leading to a taxonomy with 288 fundamentally different classes of philosophy. The relevance of a 
categorization of philosophies to the organization of objectives for research is that the philosophies represent views on the possible character and purpose of the knowledge that results from the research, so the research is an activity performed to discover knowledge, potentially of any of the possible kinds.

Varro's classification of possible philosophies has been transformed into a classification of research objectives, Table 14.1. The "Life Goal" dimension which expresses a view on the relation between the knower and knowledge may, at first, be criticized as redundant, since it seems similar to the "Knowledge Goal" dimension with the effect that the factors $\{K 1, K 2, K 3\}$ and $\{L 1, L 2, L 3\}$ appear not orthogonal. The distinction of these dimensions is in the weltanschauung of the researcher. The "Knowledge Goal" dimension concerns the rational description of the research project purpose whilst the "Life Goal" dimension concerns the existential purpose of knowledge and research in general. The other dimensions are distinct and distinguish characteristics of the possible purposes of research. Thus, in the research traditions of a field the "Knowledge Goal" dimension leads to the articulated description of what is considered research and how it should be done. The "Life Goal" dimension leads to the mores of the social community of the field, in which the research is done. 
Table 14.1 Taxonomy of research objectives built from Varro's classification of philosophies.

\begin{tabular}{|c|c|c|}
\hline Dimension & Code & Possible categories \\
\hline \multirow[t]{3}{*}{ Desired Outcome } & $D 1$ & Theoretical development of the field \\
\hline & D2 & Practical development in the field \\
\hline & D3 & Development of theory and practice \\
\hline \multirow{3}{*}{ Knowledge Goal } & K1 & Goal is knowledge \\
\hline & $K 2$ & Goal is the field of application \\
\hline & K3 & Goal is both knowledge and application \\
\hline \multirow[t]{2}{*}{ Beneficiary } & $B 1$ & Investigator \\
\hline & $B 2$ & Others in the community \\
\hline \multirow[t]{2}{*}{ Certainty } & $C 1$ & Knowledge is definite and certain \\
\hline & $C 2$ & Knowledge is contingent \\
\hline \multirow[t]{2}{*}{ Tradition } & $T 1$ & Build within the tradition of the field \\
\hline & $T 2$ & Challenge, replace or reject the tradition of the field \\
\hline \multirow[t]{3}{*}{ Life Goal } & L1 & Enjoy knowing \\
\hline & $L 2$ & Enjoy practising \\
\hline & L3 & $\begin{array}{l}\text { Enjoy knowing and } \\
\text { practising }\end{array}$ \\
\hline
\end{tabular}

The dimensions in the taxonomy of Table 14.1 are:

Desired Outcome, $\{D 1, D 2, D 3\}$, concerns the researcher's belief about the objective of the research project. The objective of a project may be to improve theory about the subject, practice related to the subject or both. Development of theory emphasizes intellectual constructs describing the framework of ideas in the field. Development of practice concerns improving ability to act appropriately. For a particular subject matter research conducted from each of these perspectives will involve different methods and have different indicators of success.

Knowledge Goal, $\{K 1, K 2, K 3\}$, concerns whether the knowledge is valued for its intrinsic or its instrumental value. This contrast divides between disciplines oriented towards creation of knowledge, such as the sciences, and those oriented towards performance of some activity or achievement of particular outcomes, such as engineering.

Beneficiary, $\{B 1, B 2\}$, is concerned with the user of the research product. The researcher performs work intended to benefit a target audience. The target audience may be the researcher, 
for research to satisfy curiosity or for self-development, or others, where the intention is dissemination. This has implications, inter alia, for the extent to which generalizability is a focus of the work.

Certainty, $\{C 1, C 2\}$, concerns the researcher's epistemological stance. Knowledge may be viewed as being true in an absolute sense, that is always true, true everywhere and the same for all knowers. Alternatively, knowledge may be viewed as relative or contingent, depending on the perspective of the observer. This dimension concerns whether it is possible to know things objectively or only experientially.

Tradition, $\{T 1, T 2\}$, concerns whether the tradition of the field is viewed as sufficient, so that the research is done to enhance knowledge of some aspect of the tradition within the overarching framework of the field. Alternatively, the iconoclastic position sets out to challenge or reject the tradition. Challenging the tradition may involve interpreting observations significantly differently than the current tradition while rejecting the tradition may be done to be different or to radically reformulate the tradition.

Life Goal, $\{\mathrm{O} 1, \mathrm{O} 2, \mathrm{O} 3\}$, concerns the researcher's existential engagement with the knowledge under development. The Objective in Life may be the pleasure obtained through knowing, or through practice and achievement, or a combination. The Desiderata dimension concerns what is regarded as innately desirable, and Relation to Knowledge concerns a rational view of the nature and purpose of knowledge while the Objective in Life concerns the researcher's relation to the knowledge.

\subsection{Discussion of Methods to Investigate Emergent Effects}

Six approaches to research that may be used to investigate questions or issues related to emergence are discussed in this section. 


\subsubsection{Case Studies}

"Case studies" is used here to refer to researcher initiated investigations motivated by the researcher's purpose of discovering the facts and relationships relevant to a case. A case study investigation is conducted by finding available materials and, possibly obtaining primary data collection from participants or measurement of the case and other similar sources. The case study method is commonly used in some fields, amongst which are investigation of engineering projects, and engineering accidents and disasters. In these investigations "emergence" is often a phenomenon that makes the particular case interesting to investigate, for example because there is something instructive about how a project progressed, or an accident occurred because of an unexpected effect of the confluence of factors, matching one of the common views of "emergence" discussed in section 2.

The investigator driven case study is limited because the study is constrained by the source materials that the participants with direct awareness of the situation choose to make available or which have otherwise been placed in the public domain. This limitation of available sources prevents assurance that the materials available present a complete and fair view of the situation, and that there is nothing that would lead to different conclusions in materials not divulged. This impacts the confidence that can be placed in any conclusions. A second major limitation of the case study method is that its subject matter is a particular case. This raises the question of whether what is found is just the description of a particular case or is, in some way, generalizable and the bounds of the generalizability. The impact of this limitation is whether the case should be read as a description of a past case or can be used to make recommendations for analogous cases. Turning from the limitations of the case study method we consider the strengths of the approach. The case study approach is well suited to addressing questions of how or why, and therefore for 
providing insight into a state of affairs (Yin 2014). If one understands the how and why relations that have been present in particular instances of a class of things, then the reasonable generalization is insight into the class of things of which the instance is an exemplar. The challenge is to recognize and define the attributes of the class appropriately so that the belief that one has gained insight is appropriately applied.

Depending on the purpose of the case study work the researcher may use a relativist, interpretivist or realist epistemology (Yin 2014). The choice of epistemological position to be used reflects a conclusion about a combination of the case, the information available about the case and the researcher's purpose in performing the investigation. We now consider the case study method in relation to Table 14.1, to determine the most likely hex-tuple descriptors of the objectives of research.

\section{$14.1\langle D 3, K 2, B 2, C 2, T 1, L 1\rangle$ for a relativist epistemology}

$14.2\langle D 2, K 2, B 2, C 2, T 1, L 1\rangle$ for an interpretivist epistemology

$14.3\langle D 1, K 2, B 2, C 2, T 1, L 2\rangle$ for a realist epistemology

Case studies are usually motivated by one of two scenarios. The first is understanding of an accident or disaster, usually on a newsworthy story scale, and in most cases the event that made for the problem was an unintended and disadvantageous emergent event. That is, case studies are usually investigations of emergence, the presence of which was discovered through the observation of unexpected or unintended effects and frequently these effects are disadvantageous. The usual purpose of the case study is to identify a crucial contributing factor in order to make a recommendation which would prevent similar problems.

\subsubsection{Forensic Case Studies}

We now turn our attention to a distinct kind of case study, the forensic case study. A forensic 
investigation is an investigation of a particular case, and always performed with a view to discovery of the cause of a problem, which may be motivated by the need to attribute blame or to formulate recommendations to improve future outcomes. The purpose of forensic case investigations may be legal or organizational. In a legal investigation the immediate purpose of the investigation is a formal investigation such as a court case or coroner's inquest.

Organizational purposes for this type of investigation are driven by a need for rigorously founded recommendations for future practice, although the rigor required may not need to be of a standard that would be required for legal proceedings.

The forensic case study situation empowers the investigator with discovery powers much stronger than those available to the "normal" case study researcher. The legal or organizational principal directing the investigation gives the investigator strong powers to demand relevant information, in contrast to the curious investigator's need to rely on the willingness of information holders to divulge. This power is particularly important in situations where information holders may be afraid of either legal action or embarrassment associated with the matter. A forensic case study, like other investigations of the past, can only obtain primary evidence, either information or tangible, created while the events under investigation were unfolding. The other evidence available to the investigator is secondary, including testimony of participants, potentially taken under oath, and remnants of the things that remain at the time the investigation was initiated.

An investigation to identify responsibility for a situation will lead to an account of events in the case. The account does not, itself, provide recommendations for the future. In contrast, a coronial inquest, commission of inquiry, or less formal intra-organization equivalents are intended to yield recommendations to improve future scenarios. The hex-tuple descriptors of the research 
objectives of these two approaches to the forensic case study are:

$14.4\langle D 2, K 1, B 2, C 1, T 1, L 2\rangle$ to discover responsibility for past events

$14.5\langle D 2, K 2, B 2, C 1, T 1, L 2\rangle$ to make recommendations to improve the future

Like the general case study, the forensic case study is performed to understand emergent effects found through events which led to a bad outcome. The forensic case study is motivated by a bad outcome whereas the general case study may be motivated by either a bad or an interesting outcome.

\subsubsection{Post Hoc Empirical Studies}

In some situations, an observer is limited to observation of things and scenarios that already exist without the opportunity to make interventions but the observer desires to form conclusions about classes of cases. The limitations on the investigator intervening may be practical, such as cost, time required, or ethical, such as investigating an effect hypothesized to cause of harm. Constraints such as these limit the investigator to observing cases that exist and seeking to form conclusions about them. Such investigations are post hoc empirical studies. The principal feature of post hoc studies is that the investigator cannot control the conditions of observations and may only have data collected by others available, with differences in observation methods for data from different sources.

In post hoc empirical investigations the researcher observes a sample of cases selected from a broader population with respect to a set of parameters that represent the researcher's model of the phenomenon of interest with the result that conclusions can be formulated within the construct of the model but it is not possible to discern if the model is a valid method to view the phenomenon (Ferris 1997). This epistemological problem represents a serious limitation of this type of research. The difficulty caused by the epistemological problem is that one cannot know if 
correlated observations have a direct causal linkage or if the correlation could be explained by some other causal mechanism. A full account of the possible causal relations of two correlated sets of observations is given by Skrabanek and McCormick (1990) Four difficulties with post hoc empirical studies are discussed here:

1. The cases studied pre-exist the study. Thus, the samples are chosen on the basis of presence of an outcome manifestation resulting in all observations of other factors being made after the action has occurred. This prevents investigation of whether the observed effect results from the matter of interest or from other known or unknown effects.

2. The cases receiving each of the treatments considered in the observations cannot be randomized because the treatments were applied by someone who believed that the treatment applied would increase the probability of achieving their desired outcome or the allocation of treatments to cases has no explanation. Observations cannot be used for determining any statistical relationship of application of a treatment to cases exhibiting particular characteristics because the characteristics that identify classes of case are not the basis for assignment of treatments. In turn, this prevents use of the results to predict the likely outcome if particular treatments are applied to a random member of the relevant class.

3. The pre-existing nature of the observed cases introduces the risk that determination of the surmised "background" factors may be biased by knowledge of the outcome class of the sample. Performing such a data collection process without observer awareness of the outcome classification of the sample is either difficult or impossible because in the post hoc observation situation the outcome exists before the initial condition is questioned.

4. Despite all attempts to the contrary by a diligent investigator the sample actually studied 
may reflect a bias towards one sub-group in the population. For example, a medical investigation of patients presenting to a clinic is biased by the self-selection factor that the people presenting had something that made them believe they needed and to seek assistance, and therefore they are not representative of the broader population. This factor can bias a doctor's perception of frequency of a particular illness (Singer et al. 1997, Skrabanek and McCormick 1990).

The post hoc study may struggle to yield insight about the emergent effects which may have motivated it. The challenge may arise at several levels: the difficulty in obtaining suitable data to make a judgment about correlation of manifestations, and absence of control for extraneous factors makes conclusions about causality difficult.

The hex-tuple descriptor of the research objectives of post hoc empirical studies is:

\section{$14.6\langle D 1, K 1, B 2, C 1, T 1, L 1\rangle$ for post hoc empirical studies}

The post hoc empirical study is a method to discover emergent effects through observation of facts on the ground but is challenged to provide causal explanations for correlations of factors and therefore presents emergence as a phenomenological matter.

\subsubsection{Experimental Empirical Investigation}

Experimental investigation is a research method which is commonly regarded as the best method for discovery of the properties of things. Where, in post hoc studies it is only possible to observe outcomes and then investigate antecedent conditions which enables discovery of predictive correlations an experimental study enables, with appropriate design, the investigation of causality. In experimental studies there are well established protocols for randomly assigning cases to treatments and, to the greatest extent possible, blinding the investigator and other participants to the correlation of cases and treatments, which is done to reduce observation bias, 
in which an observer may report outcomes they expect rather than what is objectively present. Emergence presents a challenge in the context of experiments. Usually an experiment is performed to find the relationship of an independent variable applied to a class of entity to one or more dependent variables observed in the experiment. This requires an a priori theory of what the investigator believes may explain the situation represented by the experiment. In most cases an experiment is designed to investigate the theory posited through testing hypotheses about what one would expect to observe if the theory were true. Note, the hypothesis is usually reconstructed as the null hypothesis, because disproving the null hypothesis is as close as one can approach demonstrating truth of the positive hypothesis. A population of the entity of interest is sampled with the purpose of avoiding bias of which treatment is applied to samples, which in turn may bias conclusions about relationships of the variables. Enough samples are subjected to the experimental conditions to enable statistical testing of the observations using accepted inferential statistical techniques. This process demands that the experimental conditions exclude the possible effect of anything on the sample other than the independent variable under test. This enables discovery of one class of emergent effect: that which regularly occurs as a result of an underlying mechanism. This is the kind of emergent effect which is embedded in the normal practice of engineering as the scientific principles which are applied in design and action. The aspects of emergence associated with the idea of surprising or unexpected effects are unlikely to be discovered through experiments because the method of performing experiments necessarily aims to separate the relationship under observation from the influence of any extraneous effect. This intentional isolation of the experiment from other effects results in physically excluding conditions in which unexpected things can be seen. If something happens in an experiment which is of the form of "surprising emergence" it is likely that the result will be 
sufficiently different than the results obtained for other cases studied in the experiment that the observation will be classified as an unexplained outlier, and ignored.

Experimental investigations are capable of producing knowledge which can be used in the "forward path" of engineering design to propose, and through analysis, demonstrate, design ideas which should produce desired emergent effects. The problem is that the design of the experimental method will not enable discovery of emergent effects other than the relationship specifically investigated in the experiment. In addition, the presuppositions about the nature of knowledge embedded in the experimental method, such as repeatability and broad applicability make it unlikely that experiments will identify unusual emergent effects because such effects, if not part of the phenomenon that the experiment is designed to investigate will not be demonstrable through inferential statistical methods.

Experiments are motivated by one of three goals. The first is the pure scientific desire for knowledge about a phenomenon because the researcher finds the phenomenon interesting but without any particular intended application of the knowledge. Second is the applied knowledge motivation in which experiments are performed to investigate a particular phenomenon for the purpose of potential application. The intended application may cause the researcher to be concerned with only a certain subset of the potential range of independent variables, or to impose other constraints on what is investigated. A third motivation is the research and development scenario in which the subject matter of the experiments is things proposed as potential designs and the goal is to determine performance under specified conditions. These three motivations map differently to the objectives of research described in Table 14.1. The hex-tuple mapping is shown in equations 14.7 to 14.9 .

14.7 $\langle D 1, K 1, B 2, C 1, T 1, L 1\rangle$ for a pure scientific motivation 
14.8 $\langle D 3, K 3, B 2, C 1, T 1, L 3\rangle$ for an applied scientific motivation

$14.9\langle D 2, K 2, B 2, C 1, T 1, L 2\rangle$ for a research and development purpose

\subsubsection{Research to Enable Engineering}

The purpose of engineering work is to perform action which enables desired outcomes to be achieved. This work uses the knowledge of the various sciences with the purpose of informing action of design and building of things that will perform as intended in a range of situations relevant to the particular design. Success in this task demands willingness to use knowledge from any field of science and any experience, codified in the best currently available expression of the knowledge in order to inform proposed design ideas, to analyze and evaluate those ideas, and to review and criticize the design ideas for unintended emergent effects. The first three verbs in the previous sentence, "propose", "analyze" and "evaluate", work with the emergent effects that are known phenomena in the relevant branches of science. In this part of the engineering task the engineer works with known emergent effects to develop desired outcomes. The latter two verbs in the same sentence, "review" and "criticize", encounter the problem of unintended emergent effects. Some of this work can be done by finding out if there is existing scientific knowledge of effects which appear in the design proposal because of the juxtaposition of elements and for which there is an existing body of relevant knowledge.

For example, electronic design at the simpler level often taught in undergraduate degrees focuses on the circuit theory properties of the arrangement of components. A practical realization of the circuit will assemble those components onto a substrate and put in a housing, and through that physical construction will introduce mechanical and thermodynamic effects which will interact with the environment of intended deployment. These factors can be analyzed by introducing knowledge from the relevant disciplines in the review process. If the engineering team challenge 
the original design proposal with the right review questions the emergent effects of these kinds of issues can be identified. The difficulty is knowing the right review questions to properly challenge the design concepts. Once the project passes the tests applied to it by the engineering team analysis of proposals the project progresses to instantiation in which various empirical tests can be performed on parts, or the whole, of the product, or experimental test-beds which represent aspects of the product. Tests may be performed to determine if design concepts are appropriate, or whether particular designed items are suitable for incorporation into the design. In most cases the test purpose is to subject the designed entity to a set of conditions and observe parameters. If the measures are consistent with design predictions, in most cases, the entity is declared to have passed the test and the process progresses to the next test. In this situation it is assumed the models used sufficiently completely describe the situation as to provide assurance the model is sufficiently accurate as to proceed with its use, and trusting its conclusions. This scenario is consistent with the use of Measures of Performance which are usually used to demonstrate compliance of the design to specification. Tests designed to determine Measures of Effectiveness, where the entity is tested under more realistic operational situations are more likely to discover unintended emergent effects and the limited resources which can be expended on such tests necessarily leave many conditions within the declared operational envelope of the entity which have not been tested. Whether an unexpected emergent effect is discovered in effectiveness tests or in use, the discovery is late enough in the lifecycle as to present a significant difficulty to the success of the project. This is the problem that research into emergence should aim to pre-empt.

Engineering projects conducted in the normal way produce both the deliverable products, and learning about what is achieved by particular designs. However, this learning is in the form of 
case specific outcomes, and does not provide a systematic foundation for future action. As each project is performed discoveries about the interaction of elements within the project, both the deliverables and the characteristics of the performing organization will be made or, at least, enabled. These will form a scatter of points in a space with no systematization to assist interpretation that will enable recommendations about future action that can be put forward with assurance.

However, learning of engineering useful knowledge about the design of systems, and therefore the emergence that occurs in the development of a system as related to both the things delivered by the project and the methods of performing the work can be learnt through appropriately designed research projects. Complex, or large scale, engineering projects of the kind that deliver systems, and therefore have the potential to inform the engineering of complex systems, are not amenable to experimental investigation for a range of reasons including resources and the impossibility of controlling to study the effect of any particular independent variable without confounding through a variety of other factors. This demands consideration of other methods which are capable of generating useful knowledge about the impact of design or method choices in such projects These methods will differ from those used in other types of investigation. Engineering relevant research to discover the emergent effects in projects related to systems can approach the investigation from several perspectives. Projects may be performed to understand how to deliver in reasonably known areas of practice, or the provision of radically new kinds of deliverables, or investigation of the method a particular engineer should use to achieve desired results. These three purposes map differently to the objectives of research described in Table 14.1. The hex-tuple mapping is shown in equations 14.10 to 14.13 .

$14.10\langle\{D 2, D 3\}, K 2, B 2, C 1, T 1, L 2\rangle$ for knowledge about deliverables in a reasonably well 
known field

14.11 $\langle\{D 2, D 3\}, K 2, B 2, C 1, T 2, L 2\rangle$ for knowledge about deliverables in a radically new field $14.12\langle\{D 2, D 3\}, K 2, B 2, C 2, T 1, L 2\rangle$ to discover methods to suit particular engineers, personalized practice

$14.13\langle\{D 2, D 3\}, K 2, B 1, C 2, T 1, L 2\rangle$ to discover methods to improve the researcher's own practice

\subsubsection{Research into Fundamental Theory}

Fundamental theory research, as distinct from research in theoretical science, operates in an abstract space, generally of mathematical representations of things that either exist, or are postulated to exist. The entities under study are abstractions rather than tangible things. The method of such research is normally mathematical and its practical impact relies on the existence of homomorphism of the mathematical constructs used in the investigation and the entities which they are used to represent. The mathematical abstraction of the investigation can identify results that would be observed if one can find a way to observe an effect, which, in turn, if observed would result in determination that the effect posited is real and, by extension, that other outcomes of the model are likely to be observed as instantiations of emergent effects.

Historically, fundamental research methods have been closely associated with theoretical physics, and similar fields, but it is reasonable to consider their use in engineering, in which the abstract space may enable the discovery of the presence of emergent effects and judicious development of experiments to test critical issues could lead to the discovery of grounds to believe in the existence of emergent effects which may be rare, or at least, not yet actually observed.

Fundamental theory research can be mapped to the objectives of research described in Table 14.1 
as described in equations 14.14 and 14.15 .

$14.14\langle D 1, K 1, B 2, C 1, T 1, L 1\rangle$ for fundamental extensions to current theory

$14.15\langle D 1, K 1, B 2, C 1, T 2, L 1\rangle$ for fundamental revision to current theory

\subsubsection{Summarization of Research Objectives}

We now collect together the hex-tuples describing the research objectives for investigations in each of the six types discussed above as relevant to, or potentially relevant to, the investigation of emergent effects in engineering and present them in Figure 4.1. In Figure 4.1 we observe that each kind of research, and each variation within each kind, involves a different combination of factors to describe the objective of the work. This shows that the objective in each case is to produce knowledge for a different purpose, and that the characteristics of the purpose vary in case specific ways, with the result that knowledge with different characteristics will be required. The effect is that different research methods are required to support the diversity of purposes. As discussed earlier in this section of the chapter, each of these research objectives and scenarios develops knowledge that in some way informs our understanding of emergence. It is not surprising that emergence can be elucidated by so many different methods of and approaches to research because emergence itself has many facets, as discussed section 14.2.

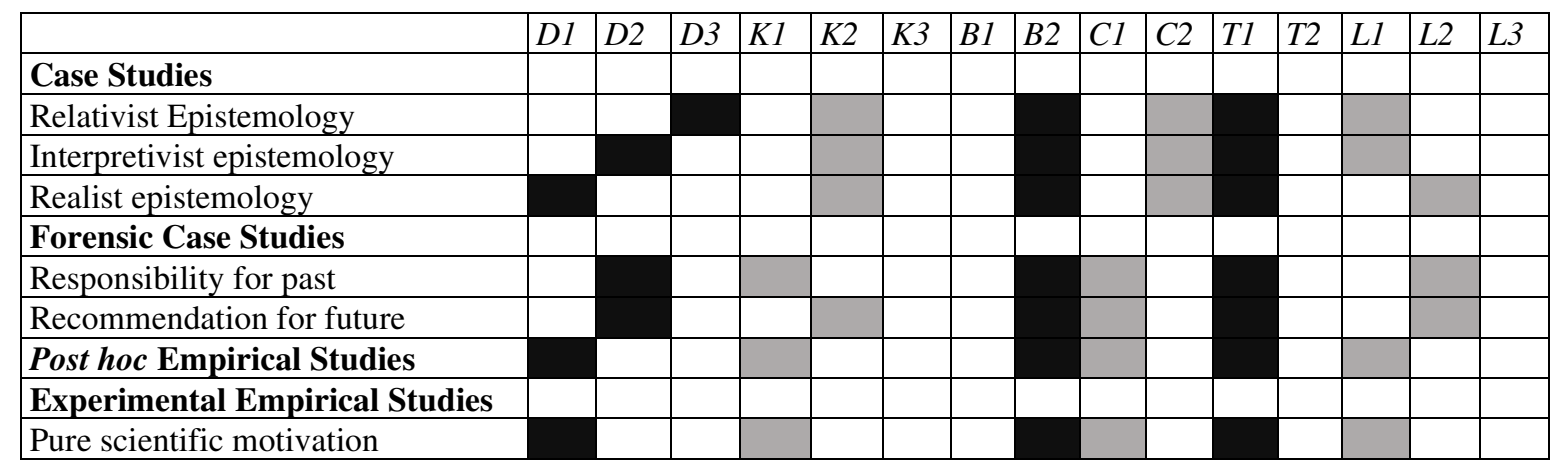




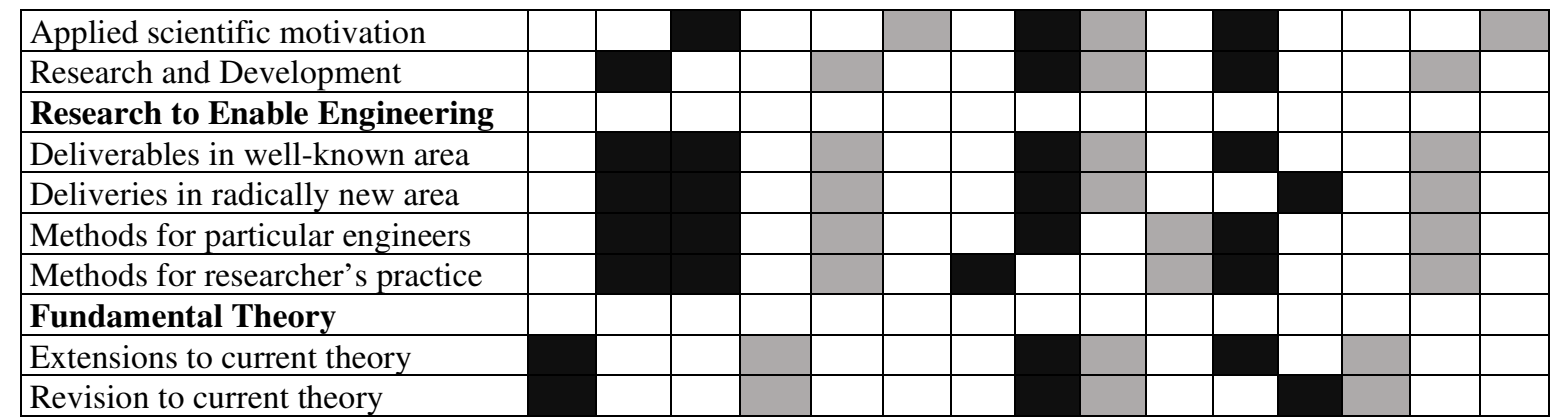

Figure 4.1. The hex-tuples describing the research objectives for various types of research that will inform understanding of emergence in a way that informs engineering practice.

\subsection{Implications for Research in Engineering: What is Considered Publishable}

In section 14.4 we outlined six research approaches often used in engineering research and investigations. The approaches are used to differing extents, and in different settings the driving motivations for research result in different emphasis on each of the types of research. In the academic community research achievement is one of the major drivers of careers, with the consequence that most academics seek to succeed in producing research publications in venues considered appropriate by their universities. A consequence of this drive to be published in the recognized venues is that the entire research activity is constructed in order to lead to recognized publications. This involves a series of steps rather like the list below:

1. Determine the intended publication venue, such as Journal X. The guidance to authors for the target journal describes the kind of papers published, which includes both the subject matter scope and, more importantly, the kind of research output that is considered suitable. 
2. The researcher designs a research project, motivated by an interest in a topic within the scope of the journal.

3. The specific research questions are framed so that the method required to find an answer will produce results of a form that fit the normal published content of the journal. The act of framing the research questions to fit the usual published content of the journal may distort the original motivation for the research. The effect is that the results reported, whilst actually answering the formal research question do not form an answer to the issues which motivated the research.

In general, in the research journals there is a strong weighting towards the publication of reports of testing of an hypothesis. The reason for this is that the concept of knowledge in classical epistemology, see section 14.1, demands justified true belief. The interpretation of "justified" in science and engineering has taken the position that justification is demonstrated through a process of positing an idea, the hypothesis, and performing an activity which tests that hypothesis. The practical effect of this is that, whilst other methods are acknowledged by many, statistical testing of observations conducted specifically with a view to testing a particular hypothesis are the most common form in research journals.

There are several causal factors:

1. A statistical hypothesis test study has clear measures of something which can be tested using established analysis methods to produce clear conclusions about support for the hypothesis. Also the process of analysis can be checked using the information included in a standard presentation of results. This characteristic provides confidence that the knowledge presented satisfies the justified true belief test (Haufe 2013). This characteristic leads to relative clarity in the review process and reduces the matters 
over which reviewers can contend, thus reducing the risk of delay or rejection.

2. If an investigation is designed to produce a statistical test of an hypothesis the investigators have a basis for a higher level of confidence from the outset that if the work is performed following standard competence recognized in the field that the manuscripts reporting the work are likely to be publishable in their target journal.

3. Since publication is understood as the indicator of successful research, grant funding agencies, which have a remit to support good research leading to scientific knowledge, are more likely to fund projects designed to test an hypothesis because it is clearer how the result is likely to be accepted as a contribution (Haufe 2013). This satisfies the grant funder's "value for money" measure. Thus, proposals for funding are most likely to be designed to fit the form that is more likely to gain funding.

4. Even if research is not supported by a grant agency the researchers will seek to publish, which biases their methodology towards the hypothesis test formulation, and their motivation for publication is further driven by personal track record of research outputs closely aligned with future funding proposals, further biasing them to perform projects that conform to the recognized format.

5. There is a current trend to "objectively" measure academic staff performance with research achievement being the primary dimension for "success". The measures applied are often of things that are countable, for two reasons. Counts of things are "objective", which removes all the complications and effort associated with any attempt to evaluate "quality", other than using a countable proxy for "quality". Initially, output measure counts of papers published were used. In response many more venues for publication, such as new journals and a multiplication of 
conferences, appeared. This led to attempts to rank venues according to whole of venue esteem measures. Then it became recognized that many papers were either never cited, or perhaps cited only in self-citation or by co-workers, so citation counts, first of total citations and later independent citation counts, and further metrics building on citation counts were developed, but still the "quality" measure was a countable proxy for "quality", not an actual measure of quality. In addition, input measures for research, primarily amount of funding received for projects that the individual proposed and "won" are also applied. These measures may be direct money amounts, or amounts weighted by the kind of funding entity, with a ranking that may favor "pure science" funding over funding closer to application and product development. Measures of success as an academic, and primarily that means as a researcher, are used in career decisions including continued employment and promotion, leading many to distort their research to fit the evaluation constructs rather than to focus on investigation that directly addresses the questions that motivated their work.

This brief account of factors driving the kind of research performed shows challenges for the performance of research into emergence. We consider the six approaches to performing research discussed in section 14.4 of this chapter. Both types of case study and the post hoc empirical approaches could be approached from the perspective of collecting and analyzing data in various ways until something interesting is found. This perspective is widely disparaged as a "fishing expedition" in which any discovery of an apparently interesting relationship is the result of stumbling into the result. Such an approach is viewed poorly by many researchers (Haufe 2013). The two case study approaches could be approached through positing an hypothesis and 
performing an investigation seeking to find support or refutation of the hypothesis. An hybrid of the two may be used if the researcher has no a priori basis for an hypothesis but the part of the work which will be better structured in reports and gain higher respect is the latter portion that works with a formulated hypothesis. Reports of the work may even be written, after the event, to be structured as if what was done was an hypothesis test.

The post hoc empirical investigation may be used to test an hypothesis. The challenge is that the lack of control of the situation limits the hypotheses to questions such as whether observed correlations evidence an underlying non-random factor, which requires considerable care to translate into a study that enables conclusions about causality.

The experimental approach is directly linked to hypothesis testing since most experiments are designed to enable testing a particular hypothesis. Setting up an experiment consumes resources and therefore, for the reasons described above, results in design that will enable testing of an hypothesis because that yields the highest probability of publication.

Research to enable engineering is concerned with enabling engineers to perform better the various kinds of work engineers perform. This includes all the contributions of engineers to any stage of the product life cycle. The goal of this work is delivery of better engineered outputs, that is things and processes in the real world. The goal is not perfecting knowledge about the effects and phenomena involved in the products and processes but, rather directly the quality and effectiveness of the things having impact in the world. Doing the work that ensures good results depends on the engineer being able to predict the effect of any design idea posited. This prediction demands reliable knowledge of the immediate effects of phenomena and also of the interactions of the elements with each other. Understanding the effects of interactions is difficult. Examples of the difficulty are the challenges around electromagnetic compatibility or the 
impossibility of complete testability of complex devices such as microprocessors because of the unmanageably large number of combinations of states in these scenarios. Engineering useful knowledge of emergence demands discovery of effective solutions to these problems and other problems with similar complexity.

In fundamental theoretical research the subject matter determines the place that any type of emergent effect has in the research. To the extent that the fundamental research investigates relationships emergence is the subject matter.

\subsection{What Research into Emergence will Help Engineering}

The existence of the phenomenon of emergence has been observed since ancient times and has been exploited in the practice of engineering throughout history. The history of the development of engineering knowledge until the rapid development of engineering and the parallel development of the sciences from the industrial revolution era was largely one of building according to known good practice with the occasional more ambitious project. From time to time an ambitious project would prove to have been too ambitious, with an ensuing disaster. The disaster often led to investigation and discovery of means to pre-empt a disaster of that class. Classically this pattern is recorded in the development of medieval cathedrals, but even in the "scientific" era of engineering, of the past century there are many similar examples. A few, randomly selected examples include the Tacoma Narrows bridge failure, the collapse of the original box girder design of the Westgate Bridge in Melbourne, the loss of the first ancestor of the B-17 which proved too much for one pilot to handle, and the loss of several aircraft attempting to break the sound barrier. In each case additional research was done and means to successfully address the causal issues were found.

Events like those listed above are what have been noted as the outcomes of what is often called 
emergence, the sub-class of emergence associated with unexpected and deleterious outcomes. Specific instances such as these prompt research efforts to discover the causes of the unexpected outcomes, motivated by the specific case, but seeking understanding that is generic enough to apply to a class of cases of which the specific case is representative. The work that investigates the particular emergent effect may follow any of the approaches described in Section 14.4. This approach has a scope limitation because the emergence investigated is specific to the particular problem space and is performed after problems have been encountered.

Another approach to investigation of emergence is to investigate emergence as a general phenomenon. This approach describes emergence in general, but at that level of generality would be significantly challenged in the possibility of developing knowledge which would be usable by engineers in performing any of the various aspects of engineering.

What is needed is research which will enable the discovery of emergent effects before the product or system is constructed. It would appear that a potential path forward, with sufficient strength to be worth pursuing, would be to investigate the integration of computer modelling used in the design analysis process. Current modelling methods largely focus on analysis tools which operate on a model of the proposed product described from the viewpoint of a traditional engineering discipline. A design idea that appears to work in one domain can then be exported to the modelling tools for other domains of engagement to determine the properties of the design idea in those other domains. The work of exporting to other modelling tools is often considerable, reducing the amount of cross domain modelling views which are performed and the cycle time at which such analyses are performed. This allows for unfortunate design mistakes to be propagated until remediation is likely to become very difficult or expensive, resulting in problems that are likely to become evident too late in the system life cycle. 
The remedy would be research that enables the integration of the technical discipline views of things under development from the perspective of all technical disciplines concerned with the development project lifecycle within a shared modelling environment. For instance, the modelling environment for a product, for example a mobile phone, would have a common database representing the circuit at the levels of circuit theory, electromagnetic radiation, electromagnetic compatibility, thermal properties, mechanical properties of physical construction, vibration, shock and impact, life cycle cost, reliability and maintenance characteristics, human factors, etc, all modelled within a single modelling environment so that where data is available for predicting effects in any of the domains, those effects can be found and used in design decisions. Where the necessary data is unavailable the absence of the data would be flagged so that a decision could be made whether to investigate a specific effect which would create the required data, or to proceed in the knowledge of the risk the uncertainty presents. Either way there would be a record of the conclusion and rationale, which would be useable in the event of an investigation.

An alternative approach, possibly as a stepping stone to the full modelling approach, may be the collection of information on all known emergent relationships in a construct that enables rapid identification of possibly relevant relationships in a structure like the TRIZ construct used to guide design conceptualization. A construct of this type would provide engineers with a manual tool that could enable review of all known emergence causing relationships. Such a construct would also assist in the design of the modelling analysis concept suggested above.

\subsection{Conclusions}

In this chapter we have discussed the nature of emergence in relation to the interests and concerns of engineers. Research that will assist engineers in the tasks of engineering must be 
focused on addressing issues arising from emergence. We then took an excursus through consideration of a classification of objectives of research because this highlights that there are a wide variety of activities and purposes which could frame research, and in turn the diversity of research objectives lead to a variety of research methods. These objectives were used to review six approaches used in research in engineering through which specific classes of research objectives were identified and the diversity of methods associated with the various objectives was discussed. Then we took a second excursus, discussing a current cultural pressure that is imposed on the nature of research performed in the broad set of fields associated with science and engineering. This excursus showed a significant tension between what is generally accepted as research and the kind of learning that is needed by engineers to support their work. All these threads were tied together in pointing the way forward with two goals for research which will provide engineers with practical benefit in relation to knowledge about emergence.

The simpler of these is research to develop a TRIZ-like construct that will guide engineers through the many possibilities of interaction of effects which could lead to emergent effects which need to be understood in the decision to proceed with a design idea. The other, more complex, goal is the development of a multi engineering discipline approach to modelling in a seamless, or even automated approach so that all design decisions can be informed by analysis of the many kinds of interaction effect, which is the physical origin of emergence. A system that performs this task must be capable of using existing data concern in relationships which exist in the system and of identifying data that is missing, which in turn can be used to develop research investigation to generate the necessary data to use the relationship.

Through discussion of the underlying nature of emergence and research it has been possible to propose a research goal which can profoundly assist engineers in the process of dealing with the 
matters arising from emergence.

\subsection{References}

Ackoff, R.L., 1979a. The future of operational research is past. The journal of the operational research society. 30, no 2, 93-104.

Ackoff, R.L., 1979b. Resurrecting the future of operational research. The journal of the operational research society. 30, no 3, 189-199.

Ackoff, R.L., 2001. OR: after the post mortem. Systems dynamics review. 17 no 4, 341-346. Augustine of Hippo, 1984. Concerning the city of God against the pagans. Harmonsdsworth, Middlesex: Penguin Books.

Biggs, J.B., 1999. Teaching for quality learning at university: What the student does. Buckingham: Society for Research into Higher Education, Open University Press. BKCASE Editorial Board, 2016. The Guide to the Systems Engineering Body of Knowledge (SEBoK); v. 1.7 [online]. R.D. Adcock (EIC). Hoboken; NJ: The Trustees of the Stevens Institute of Technology. BKCASE is managed and maintained by the Stevens Institute of Technology Systems Engineering Research Center; the International Council on Systems Engineering; and the IEEE. Available from: www.sebokwiki.org. [Accessed 20 Mar 2017]. Bloom, B.S., Engelhart, M.D., Furst, E.J., Hill, W.H., and Krathwohl, D.R., 1979. Taxonomy of educational objectives the classification of educational goals handbook I: cognitive domain. London: Longman Group Ltd.

Bucciarelli, L.L., 2003. Engineering philosophy. Delft, Netherlands: DUP Satellite.

Cripps, S.C., 1991. Old fashioned remedies for GaAsFET power amplifier designers. IEEE society on microwave theory and techniques newsletter. no 129, 13-17.

Ferris, T.L.J., 1997. The concept of leap in measurement interpretation. Measurement. 21, no 4, 
137-146.

Ferris, T.L.J., 2007a. Some early history of systems engineering - 1950's in IRE publications (part 1): the problem. In: 17th International Symposium Systems Engineering: key to intelligent enterprises. San Diego: International Council on Systems Engineering.

Ferris, T.L.J., 2007b. Some early history of systems engineering - 1950's in IRE publications (part 2): the solution. In: 17th International Symposium Systems Engineering: key to intelligent enterprises. San Diego: International Council on Systems Engineering.

Ferris, T.L.J., 2008. Early History of Systems Engineering (Part 3) - 1950’s in Various Engineering Sources. In: INCOSE International Symposium 2008. Utrecht: International Council on Systems Engineering.

Ferris, T.L.J., 2010. Bloom's Taxonomy of Educational Objectives: A Psychomotor Skills Extension for Engineering and Science Education. International Journal of Engineering Education. 26, no 3, 699-707.

Ferris, T.L.J., Cook, S.C., and Honour, E.C., 2005. Towards a structure for systems engineering research. In: 15th Annual International Symposium INCOSE 2005 Systems Engineering: Bridging Industry, Government and Academia. Rochester, New York: International Council on Systems Engineering: Paper 6.1.1.

Ferris, T.L.J., Cook, S.C., and Sitnikova, E., 2007. Research methods for systems engineering. In: 13th ANZSYS conference Systemic development local solutions in a global environment. Auckland, New Zealand.

Gettier, E.L., 1967. Is justified true belief knowledge? In: A.P. Griffiths, ed. Knowledge and belief. London: Oxford University Press, 144-146.

Goodman, N., 1973. Fact, fiction, and forecast. Indianapolis, Indiana: The Bobbs-Merrill 
Company, Inc.

Haufe, C., 2013. Why do funding agencies favor hypothesis testing? Studies in history and philosophy of science. 44: 363-374.

Hickey Jr, A.E., 1960. The systems approach: can engineers use the scientific method? IRE transactions on engineering management. 7, no 2, 72-80.

Hintikka, J., 1970. Knowledge, belief, and logical consequence. Ajatus, 32: 32-47.

Hitchins, D.K., 1992. Putting systems to work. Chichester, New York: John Wiley and Sons. Kline, S.J., 1995. Conceptual foundations for multidisciplinary thinking. California: Stanford University Press.

Krathwohl, D.R., Bloom, B.S., and Masia, B.B., 1964. Taxonomy of educational objectives the classification of educational goals handbook II: affective domain. New York: David McKay Company, Inc.

Nissen, M.E., 2006. Harnessing knowledge dynamics: principled organizational knowing \& learning. Hershey, Pennsylvania: IRM Press.

Ryle, G., 1948. Knowing how and knowing that. Proceedings of the Aristotelian society. 46: 116.

Singer, A.J., Homan, C.S., Stark, M.J., Werblud, M.C., Thode, Henry C, J., and Hollander, J.E., 1997. Comparison of types of research articles in emergency medicine and non-emergency medicine journals. Academic emergency medicine. 4, no 12, 1153-1158.

Skrabanek, P. and McCormick, J., 1990. Follies \& fallacies in medicine. Buffalo, New York: Prometheus Books.

Yin, R.K., 2014. Case study research design and methods. Los Angeles: Sage Publications. 